\title{
A Sliding Mode LCO Regulation Strategy for Dual-Parallel Underactuated UAV Systems Using Synthetic Jet Actuators
}

\author{
N. Ramos-Pedroza, W. MacKunis, and M. Reyhanoglu \\ Department of Physical Sciences, Embry-Riddle Aeronautical University, Daytona Beach, FL 32114, USA \\ Correspondence should be addressed to M. Reyhanoglu; reyhanom@erau.edu
}

Received 11 August 2015; Accepted 8 October 2015

Academic Editor: Ranjan Ganguli

Copyright ( 2015 N. Ramos-Pedroza et al. This is an open access article distributed under the Creative Commons Attribution License, which permits unrestricted use, distribution, and reproduction in any medium, provided the original work is properly cited.

A sliding mode control- (SMC-) based limit cycle oscillation (LCO) regulation method is presented, which achieves asymptotic LCO suppression for UAVs using synthetic jet actuators (SJAs). With a focus on applications involving small UAVs with limited onboard computational resources, the controller is designed with a simplistic structure, requiring no adaptive laws, function approximators, or complex calculations in the control loop. The control law is rigorously proven to achieve asymptotic regulation of both pitching and plunging displacements for a class of systems in a dual-parallel underactuated form, where a single scalar control signal simultaneously affects two states. Since dual-parallel underactuated systems cannot be expressed in a strict feedback or cascade form, standard backstepping-based control techniques cannot be applied. This difficulty is mitigated through careful algebraic manipulation in the regulation error system development, along with innovative design of the sliding surface. A detailed model of the UAV LCO dynamics is utilized, and a rigorous analysis is provided to prove asymptotic regulation of the pitching and plunging displacements. Numerical simulation results are provided to demonstrate the performance of the control law.

\section{Introduction}

Limit cycle oscillation (LCO) (flutter) is a self-excited aeroelastic phenomenon that can create difficulties in aircraft tracking control and could potentially result in structural damage and even catastrophic failures. Even in the low Reynolds number (low-Re) regimes, characteristic of small unmanned aerial vehicle (UAV) systems, LCO can be adversarial in flight control systems and can lead to unsafe UAV operating conditions [1]. Motivated by these challenges, automatic control methods for LCO regulation systems have been widely investigated in recent controls literature [25]. LCO suppression control systems are usually designed using moving deflection surfaces (e.g., elevators, rudders, and ailerons) to deliver the required control force or moment. However, for applications involving smaller, lighter weight UAVs, the use of heavy mechanical actuators might not be practical. As these practical considerations motivate the need for smaller, low power-consumptive control actuators, synthetic jet actuators (SJAs) have emerged as a popular alternative to mechanical deflection surfaces for UAV control applications [1, 6-14].

SJAs are practical tools for UAV LCO suppression control systems due to their low cost, small size, and low power consumption properties. SJAs utilize a vibrating diaphragm to create trains of vortices (or jets) of air through the periodic ejection and suction of air through a small orifice (see Figure 1). The resulting trains of air vortices impart linear momentum to a flow system, and this momentum transfer enables SJAs to deliver an equivalent control force or moment when implemented in UAV wings. Since SJAs only use the surrounding air of the flow system to generate the vortices, they can deliver a control force or moment with zero net mass injection across the flow boundary. This is a key benefit in small UAV applications: SJAs do not require space for a fuel supply. The boundary layer flow field near the surface of a UAV wing can be altered by using the trains of air vortices generated by the SJAs. By utilizing this SJA-commanded modification of the boundary layer flow field, SJAs can be employed to achieve automatic regulation control of LCO 


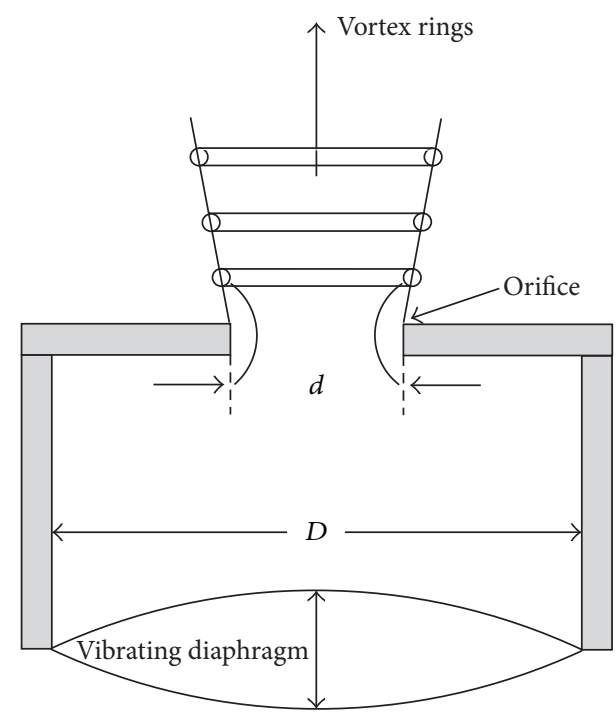

FIGURE 1: Schematic layout of a synthetic jet actuator.

in UAV wings. SJA-based control design is complicated, however, due to the inherent nonlinearity in the equations governing the SJA dynamics. Specifically, the virtual surface deflection delivered by the SJAs is a nonlinear (nonaffine) function of the voltage signal commanded to the SJAs. An additional challenge in control design using SJAs is that the nonlinear actuator dynamic model includes parametric uncertainty. This SJA nonlinear SJA dynamic model was determined empirically through repeated experiments, and it is well-accepted in SJA-based control literature (e.g., see $[10,12,14])$.

In addition to the control design challenges involved in compensating for actuator uncertainties, significant challenges arise in situations where multiple actuators lose control effectiveness. When the number of control actuators is less than the number of degrees of freedom to be controlled, the system becomes underactuated, and control design for underactuated systems presents a nontrivial challenge. The integrator backstepping technique is often utilized to address the challenge of control design for underactuated systems [15]. However, the backstepping-based control design approach can only be applied to systems in strict feedback or cascade form. Backstepping techniques cannot be utilized for systems in a dual-parallel underactuated form, where a single scalar control input simultaneously affects two states. Several open problems remain in control design for dualparallel underactuated systems.

To compensate for the nonlinearity and parametric uncertainty inherent in the SJA dynamic model, recent approaches typically employ adaptive parameter estimation, neural networks (NN), fuzzy logic rule sets, or complex fluid dynamics computations in the feedback loop (e.g., see [10, $14,16])$. These types of SJA-based control design approaches have been shown to achieve good closed-loop performance; however, control designs involving complex calculations or function approximation methods can incur an increased computational requirement, which might not be practical for applications involving small UAVs. An adaptive inverse control method is presented in [10, 14], which achieves asymptotic trajectory tracking for an aircraft dynamic model equipped with SJAs. To achieve the results in $[10,14]$, a series of adaptive parameter estimation laws are utilized along with rigorous Lyapunov-based stability analyses. Inspired by these results and motivated by the desire to investigate a more computationally minimal control strategy, our previous result in [17] was the first SJA-based tracking control approach to achieve asymptotic trajectory tracking for an aircraft using a simple (single-loop) feedback control law without the use of parameter adaptation or function approximators in the control law. A question that remains to be answered is as follows: Can a computationally minimal nonlinear SJA-based control law achieve asymptotic regulation of LCO for UAV systems in a dual-parallel underactuated form?

The contribution of this paper is the development and rigorous analysis of a sliding mode control law, which achieves asymptotic regulation of both pitching and plunging LCO in UAV wings using a single, scalar SJA as the control input (i.e., a dual-parallel underactuated system). To the best of the authors' knowledge, this is the first SJA-based nonlinear control result to rigorously prove asymptotic regulation of both pitching and plunging LCO states in a dualparallel underactuated UAV system. Moreover, to address a practical UAV scenario where onboard computational resources are limited, the result presented here compensates for the inherent SJA actuator nonlinearity and parametric uncertainty without the use of adaptive laws or function approximators. To achieve the result, a sliding mode control strategy is utilized, which employs a periodic switching law. A detailed mathematical model of the UAV dynamics is utilized to develop the regulation error dynamics, and a rigorous Lyapunov-based stability analysis is presented to prove asymptotic regulation of the pitching and plunging displacements. Numerical simulation results are also provided to complement the theoretical development.

\section{Dynamic Model and Properties}

The equation describing dynamics of LCO in an airfoil can be expressed as [18]

$$
M_{s} \ddot{p}+C_{s} \dot{p}+F(p) p=\left[\begin{array}{c}
-L \\
M
\end{array}\right],
$$

where the coefficients $M_{s}, C_{s} \in \mathbb{R}^{2 \times 2}$ denote the structural mass and damping matrices and $F(p) \in \mathbb{R}^{2 \times 2}$ is a nonlinear stiffness matrix. In (1), $p(t) \triangleq[h(t) \alpha(t)]^{T} \in \mathbb{R}^{2}$, where $h(t), \alpha(t) \in \mathbb{R}$ denote the plunging and pitching displacements, respectively. Figure 2 illustrates the pitching and plunging displacements in a standard airfoil.

Also in (1), the structural linear mass matrix $M_{s}$ is defined as [18]

$$
M_{s}=\left[\begin{array}{cc}
m & m x_{\alpha} b \\
m x_{\alpha} b & I_{\alpha}
\end{array}\right],
$$




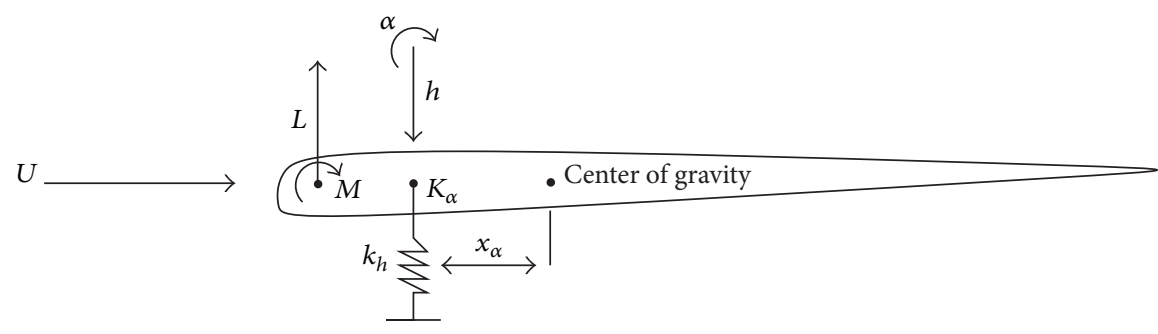

FIGURE 2: Illustration of pitch $(\alpha)$ and plunge $(h)$ in an airfoil, including lift $(L)$ and moment $(M)$ definitions and the nonlinear stiffness coefficient $\left(K_{\alpha}\right)$.

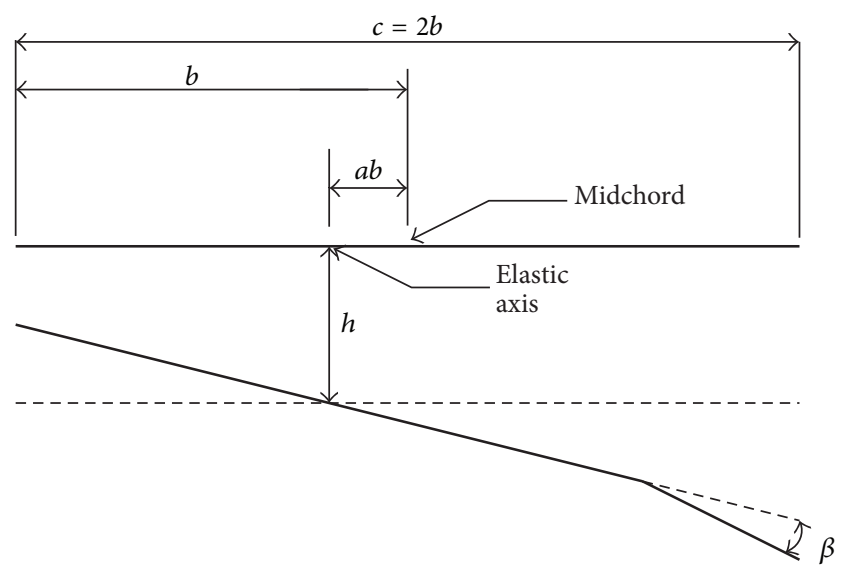

FIGURE 3: Diagram of the wing section, showing geometric parameters and the deflection angle $\beta[18]$.

where the parameter $x_{\alpha} \in \mathbb{R}$ denotes the nondimensional distance measured from the elastic axis to the center of mass, $b \in \mathbb{R}$ is the semichord of the wing [m], $m \in \mathbb{R}$ is the mass of the wing section, and $I_{\alpha} \in \mathbb{R}$ is the mass moment of inertia of the wing about the elastic axis (see Figures 2 and 3).

The structural linear damping matrix is described as

$$
C_{s}=\left[\begin{array}{cc}
C_{h} & 0 \\
0 & C_{\alpha}
\end{array}\right]
$$

where the parameters $C_{h}, C_{\alpha} \in \mathbb{R}$ are the structural damping coefficient in plunge due to viscous damping $[\mathrm{kg} / \mathrm{s}]$ and structural damping coefficient in pitch due to viscous damping $\left[\left(\mathrm{kg} \cdot \mathrm{m}^{2}\right) / \mathrm{s}\right]$, respectively. The nonlinear stiffness matrix utilized in this study is

$$
F(p)=\left[\begin{array}{cc}
K_{h} & 0 \\
0 & K_{\alpha}
\end{array}\right]
$$

where $K_{h} \in \mathbb{R}$ is the structural spring constant in plunge $[\mathrm{N} / \mathrm{m}]$ and $K_{\alpha} \in \mathbb{R}$ in $[(\mathrm{N} \cdot \mathrm{m}) / \mathrm{rad}]$ is the torsion stiffness coefficient described in terms of a polynomial as

$$
\begin{aligned}
K_{\alpha} & =2.82\left(1-22.1 \alpha+1315.5 \alpha^{2}-8580 \alpha^{3}\right. \\
& \left.+17289.7 \alpha^{4}\right) .
\end{aligned}
$$

The right hand side of (1) is explicitly given by [18]

$$
\begin{aligned}
L= & \rho U^{2} s_{p} b c_{l_{\alpha}}\left[\alpha+\frac{\dot{h}}{b}+\left(\frac{1}{2}-a\right) b \frac{\dot{\alpha}}{U}\right]+\rho U^{2} s_{p} b c_{l_{\beta}} \beta, \\
M= & \rho U^{2} s_{p} b^{2} c_{m_{\alpha}}\left[\alpha+\frac{\dot{h}}{b}+\left(\frac{1}{2}-a\right) b \frac{\dot{\alpha}}{U}\right] \\
& +\rho U^{2} s_{p} b^{2} c_{m_{\beta}} \beta,
\end{aligned}
$$

where $U \in \mathbb{R}$ is the velocity $[\mathrm{m} / \mathrm{s}], \rho$ is the density of air $\left[\mathrm{kg} / \mathrm{m}^{3}\right], s_{p}$ is the wing span $[\mathrm{m}], c_{l_{\alpha}}$ is the lift coefficient per angle of attack, $c_{m_{\alpha}}$ is the moment coefficient per control surface deflection, $c_{l_{\beta}}$ is the lift coefficient per control surface deflection, $c_{m_{\beta}}$ is the moment coefficient per control surface deflection, and $a$ is the nondimensional distance from the midchord to elastic axis. The term $\beta$ denotes the surface deflection angle of the wing (see Figure 3). By rearranging (1), the dynamics equations can be expressed as

$$
\dot{x}=A(x) x+B \beta,
$$

where $x(t) \triangleq\left[\begin{array}{llll}x_{1}(t) & x_{2}(t) & x_{3}(t) & x_{4}(t)\end{array}\right]^{T} \in \mathbb{R}^{4}$ is the state vector with $x_{1}(t) \triangleq h(t), x_{2}(t) \triangleq \alpha(t), x_{3}(t) \triangleq \dot{h}(t)$, and $x_{4}(t) \triangleq \dot{\alpha}(t)$. In (7), $A(x) \in \mathbb{R}^{4 \times 4}$ is a nonlinear state matrix (containing nonlinearities due to the torsion stiffness coefficient introduced in (5)), and $B \in \mathbb{R}^{4 \times 1}$ is the control input gain matrix. By separating the constant elements from the state-dependent elements of the matrix $A(x)$, the dynamic model (7) can be rewritten as

$$
\dot{x}=\left[\begin{array}{cccc}
0 & 0 & 1 & 0 \\
0 & 0 & 0 & 1 \\
a_{1} & a_{2} & a_{3} & a_{4} \\
c_{1} & c_{2} & c_{3} & c_{4}
\end{array}\right] x+F\left(x_{2}\right)+\left[\begin{array}{l}
0 \\
0 \\
b_{1} \\
b_{2}
\end{array}\right] u,
$$

where $a_{1}, \ldots, a_{4} \in \mathbb{R}$ and $c_{1}, \ldots, c_{4} \in \mathbb{R}$ denote known constant parameters, which are dependent on the physical parameters of the wing section. The explicit definitions of the constant parameters are unwieldy and are omitted here for brevity. In (8), $u(t) \in \mathbb{R}$ represents the virtual surface deflection delivered by the SJA (or SJA array) (i.e., the term $\beta$ 
introduced in (7)). Also in (8), the nonlinear vector function $F\left(x_{2}\right)$ is defined in terms of the stiffness coefficient $K_{\alpha}$ as

$$
F\left(x_{2}\right)=\left[\begin{array}{c}
0 \\
0 \\
\frac{-K_{\alpha}}{m\left(x_{a} b-I_{\alpha} /\left(m x_{a} b\right)\right)} \\
\frac{\left(1 /\left(x_{a} b\right)\right) K_{\alpha}}{m\left(x_{a} b-I_{\alpha} /\left(m x_{a} b\right)\right)}
\end{array}\right] \alpha .
$$

The constant control input gain terms $b_{1}, b_{2} \in \mathbb{R}$ introduced in (8) are explicitly defined as

$$
\begin{aligned}
& b_{1}=\frac{\rho v^{2} b^{2} c_{m \beta} s_{p}+\left(I_{\alpha} /\left(m x_{a} b\right)\right) \rho v^{2} b c_{l \beta} s_{p}}{m\left(x_{a} b-I_{\alpha} /\left(m x_{a} b\right)\right)}, \\
& b_{2}=\frac{-\rho v^{2} b c_{l \beta} s_{p}-\left(1 /\left(x_{a} b\right)\right) \rho v^{2} b^{2} c_{m \beta} s_{p}}{m\left(x_{a} b-I_{\alpha} /\left(m x_{a} b\right)\right)} .
\end{aligned}
$$

\section{SJA Dynamics}

The dynamics of the SJA are nonlinear and they contain parametric uncertainty. Figure 1 represents the basic structure of a SJA $[10,14]$. This SJA has a piezoelectrically driven diaphragm in its cavity that generates time varying pressure gradients across a small orifice. The periodic ingestion and expulsion of the air through the small orifice results in the formation of vortex rings which formulates a steady turbulent jet of air.

Based on empirical data, the dynamics of a SJA can be expressed as $[10,14]$

$$
u=\theta_{2}^{*}-\frac{\theta_{1}^{*}}{v}
$$

where $u(t) \in \mathbb{R}$ denotes the virtual airfoil surface deflection (i.e., the control input), $v(t)=A_{p p i}^{2}(t) \in \mathbb{R}$ denotes the peakto-peak SJA voltage, and $\theta_{1}^{*}, \theta_{2}^{*} \in \mathbb{R}$ are uncertain physical parameters.

To compensate for the SJA nonlinearity and parametric uncertainty in (11), a robust inverse control design structure will be utilized for the voltage input signal $v(t)$ [17]. The robust inverse controller can be expressed as

$$
v(t)=\frac{\widehat{\theta}_{1}}{\widehat{\theta}_{2}-u_{d}},
$$

where $\widehat{\theta}_{1}, \widehat{\theta}_{2} \in \mathbb{R}$ denote constant feedforward estimates of the uncertain parameters $\theta_{1}^{*}$ and $\theta_{2}^{*}$; and $u_{d}(t) \in \mathbb{R}$ is a subsequently defined auxiliary control signal.

\section{Control Development}

The objective is to design the scalar control signal $u_{d}(t)$ to asymptotically regulate the plunging and pitching dynamics (i.e., $h(t)$ and $\alpha(t))$ to zero. By leveraging the result in [19], $u_{d}(t)$ will be designed using a sliding mode control law with a periodic switching function as

$$
\begin{aligned}
& u_{d} \\
& =M_{0} \tanh \left\{\sin \left[\frac{\pi}{\epsilon}\left(s(t)+\lambda \int_{0}^{t} \tanh (s(\tau)) d \tau\right)\right]\right\} .
\end{aligned}
$$

Based on the dynamic equations in (8) and the subsequent stability analysis, the sliding surface $s(t) \in \mathbb{R}$ in (13) is designed as

$$
s(x)=-\frac{K_{\alpha}}{d} x_{2}+k_{1} x_{1}+k_{3} x_{3}+\sum_{i=1}^{4} a_{i} x_{i}, \quad i=1, \ldots, 4,
$$

where $k_{1}, k_{3} \in \mathbb{R}$ are positive constant control gains and $a_{1}, \ldots, a_{4}$ are introduced in (8).

\subsection{Stability Analysis}

Theorem 1. The robust control law in (13) ensures asymptotic convergence to the sliding manifold $s(x)=0$.

Proof. Proof of Theorem 1 can be found in [19] and is omitted here to avoid distraction from the main contribution of the current result.

Theorem 2 (main result). Convergence to the sliding manifold $s(x)=0$ results in asymptotic regulation of both pitching and plunging displacements in the sense that

$$
\begin{aligned}
& s(x) \longrightarrow 0 \Longrightarrow h(t), \\
& \alpha(t) \longrightarrow 0
\end{aligned}
$$

as $t \rightarrow \infty$.

Proof (plunging regulation). It follows directly from the definition of the sliding surface $s(x)$ in (14) and the LCO dynamic equations in (8) that

$$
\begin{aligned}
& s(x) \longrightarrow 0 \Longrightarrow \\
& \dot{x}_{3} \longrightarrow-k_{1} x_{1}-k_{3} x_{3} .
\end{aligned}
$$

By using the state definition $x_{3}(t) \triangleq \dot{x}_{1}(t)$, the expression in (16) can be used to show that, on the sliding manifold $s(x)=$ 0 , the $x_{1}(t)$ dynamics are governed by

$$
\ddot{x}_{1}+k_{3} \dot{x}_{1}+k_{1} x_{1}=0 \text {, }
$$

where $k_{1}, k_{3}$ are introduced in (14). Since $k_{1}, k_{3}>0$, the ODE in (17) is Hurwitz, and (17) can be used to prove that $x_{1}(t) \triangleq h(t) \rightarrow 0$ and $x_{3}(t) \triangleq \dot{h}(t) \rightarrow 0$. Thus, convergence to the sliding manifold $s(x)=0$ directly results in asymptotic (exponential) regulation of the plunging displacement $h(t)$ to zero.

Proof (pitching regulation). By substituting (14) into (8) and using the fact that $x_{1}(t), x_{3}(t) \rightarrow 0$, it can be shown that 
convergence to the sliding manifold $s(x)=0$ results in the pitching dynamics

$$
\dot{x}_{4}-c_{2} x_{2}-c_{4} x_{4}-\frac{d_{2}}{d} K_{\alpha} x_{2}=0,
$$

where $c_{2}$ and $c_{4}$ are known constant parameters introduced in (8); $d_{2} \triangleq 1 /\left(x_{a} b\right), d \triangleq m\left(x_{a} b-\mathrm{I}_{\alpha} /\left(m x_{a} b\right)\right)$; and $K_{\alpha}$ is defined in (5). By using the state definition $x_{4}(t) \triangleq \dot{x}_{2}(t)$, the expression in (18) can be rewritten as the second-order nonlinear ODE

$$
\ddot{x}_{2}-c_{4} \dot{x}_{2}-c_{2} x_{2}-\frac{d_{2}}{d} K_{\alpha} x_{2}=0
$$

After utilizing the definition of $K_{\alpha}$ given in (5), the ODE in (19) can be expressed in the form [20]

$$
\ddot{x}_{2}+b\left(\dot{x}_{2}\right)+c\left(x_{2}\right)=0 .
$$

Noting that $c_{4}<0$, the auxiliary functions $b\left(\dot{x}_{2}\right), c\left(x_{2}\right) \in \mathbb{R}$ in (20) are explicitly defined as

$$
\begin{aligned}
b\left(\dot{x}_{2}\right) \triangleq & \left|c_{4}\right| \dot{x}_{2}, \\
c\left(x_{2}\right) \triangleq & -\frac{d_{2}}{d} 17289.7 x_{2}^{5}+\frac{d_{2}}{d} 8580 x_{2}^{4}-\frac{d_{2}}{d} 1315.5 x_{2}^{3} \\
& +\frac{d_{2}}{d} 22.1 x_{2}^{2}-\left(\frac{d_{2}}{d} 2.82+c_{2}\right) x_{2} .
\end{aligned}
$$

Note. It can be shown that $c\left(x_{2}\right)=0 \Rightarrow x_{2}(t)=0$. The expressions in (21) and (22) satisfy the following properties:

$$
\begin{array}{ll}
\dot{x}_{2} b\left(\dot{x}_{2}\right)>0 & \text { for } \dot{x}_{2} \in \mathbb{R}-\{0\}, \\
x_{2} c\left(x_{2}\right)>0 & \text { for } x_{2} \in \mathbb{R}-\{0\} .
\end{array}
$$

To prove asymptotic regulation of the pitching displacement $x_{2}(t)$ to zero, consider the positive definition function (i.e., Lyapunov function candidate) [20]

$$
V=\frac{1}{2} \dot{x}_{2}^{2}+\int_{0}^{x_{2}} c(\xi) d \xi
$$

After taking the time derivative of (25) and utilizing (20), $\dot{V}(t)$ can be expressed as

$$
\begin{aligned}
\dot{V} & =\dot{x}_{2}\left(-b\left(\dot{x}_{2}\right)-c\left(x_{2}\right)\right)+c\left(x_{2}\right) \dot{x}_{2} \\
& =-\dot{x}_{2} b\left(\dot{x}_{2}\right) .
\end{aligned}
$$

The Lyapunov derivative in (26) is negative semidefinite based on inequality (23). We now use LaSalle's invariance principle to state that $\dot{V}(t) \rightarrow 0$ as $t \rightarrow \infty \Rightarrow \dot{x}_{2}(t) \rightarrow 0$ as $t \rightarrow \infty$, and thus, $\ddot{x}_{2}(t) \rightarrow 0$ as $t \rightarrow \infty$. It further follows that $b\left(\dot{x}_{2}\right) \rightarrow 0$ as $t \rightarrow \infty$ from (21). Since $b\left(\dot{x}_{2}\right), \ddot{x}_{2}(t) \rightarrow 0$ as $t \rightarrow \infty,(20)$ can be used along with property (24) to prove that $x_{2}(t) \rightarrow 0$ as $t \rightarrow \infty$.
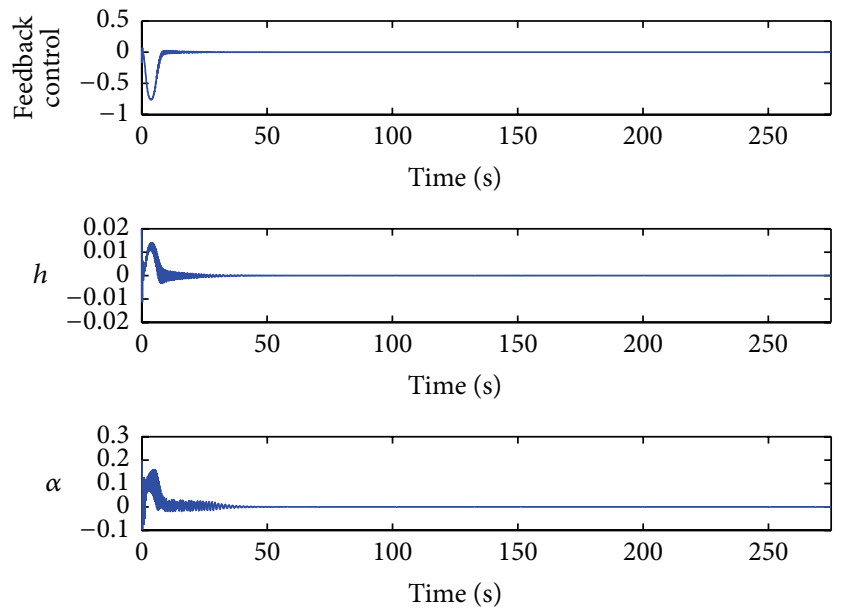

Figure 4: Case 1: $x(0)=[0.02,0.2,0,0]^{T}$; time evolution of the control input, $u(t)$, regulation error for plunging, $h(t)$ in $[\mathrm{m}]$, and regulation error for pitching, $\alpha(t)$ in [rad].

TABLE 1: Manually selected gains for robust control.

\begin{tabular}{ll}
\hline$k_{s}=9.0$ & $\epsilon=100$ \\
$M_{0}=1.0$ & $\lambda=17.0$ \\
\hline
\end{tabular}

TABLE 2: Constant parameters.

\begin{tabular}{lcc}
\hline$\rho=1.225 \mathrm{~kg} / \mathrm{m}^{3}$ & $a=-0.6$ & $c_{m_{\alpha}}=-0.635$ \\
$m=12.387 \mathrm{~kg}$ & $b=0.125 \mathrm{~m}$ & $v=13 \mathrm{~m} / \mathrm{s}$ \\
$C_{\alpha}=0.036\left(\mathrm{~kg} \cdot \mathrm{m}^{2}\right) / \mathrm{s}$ & $c_{l_{\beta}}=3.358$ & $s_{p}=0.6 \mathrm{~m}$ \\
$I_{\alpha}=0.065 \mathrm{~kg} \cdot \mathrm{m}$ & $C_{h}=27.43 \mathrm{~kg} / \mathrm{s}$ & $c_{l_{\alpha}}=6.28$ \\
$K_{h}=2844.4 \mathrm{~N} / \mathrm{m}$ & $c_{m_{\beta}}=-0.635$ & $x_{a}=0.2847$ \\
\hline
\end{tabular}

4.2. Results. In this section, a numerical simulation was created to demonstrate the performance of the control law developed in (13) and (14). The simulation is based on the dynamic model given in (1). The dynamic parameters utilized in the simulation are summarized in Table 2 and were obtained from [18].

The control gains $k_{s}, \epsilon, M_{0}$, and $\lambda$ were manually selected as described in Table 1 . The actual SJA parameters $\theta_{2}^{*}$ and $\theta_{1}^{*}$ and estimates $\widehat{\theta}_{2}$ and $\widehat{\theta}_{1}$ were selected as 32.9 and 14.7, respectively.

The control law was simulated for three different cases of initial conditions: Case 1: $x(0)=\left[\begin{array}{cccc}0.02 & 0.2 & 0 & 0\end{array}\right]^{T}$ (Figures 4 and 5), Case 2: $x(0)=\left[\begin{array}{llll}0 & 0 & 0.05 & 0.5\end{array}\right]^{T}$ (Figures 6 and 7), and Case 3: $x(0)=\left[\begin{array}{llll}0.02 & 0.2 & 0.03 & 0.3\end{array}\right]^{T}$ (Figures 8 and 9). Figures 4,6 , and 8 show the time evolution of the pitching and plunging displacements $\alpha(t)$ and $h(t)$ during closed-loop controller operation for Cases 1, 2, and 3, demonstrating the rapid convergence of the pitching and plunging displacements to zero. In addition, Figures 5, 7, and 9 show that the pitching and plunging velocities $\dot{\alpha}(t)$ and $\dot{h}(t)$ converge to zero rapidly during closed-loop operation. Further, the top plots in Figures 4, 6, and 8 show the control effort $u(t)$ used during closed-loop controller for Cases 1, 2, and 3. 

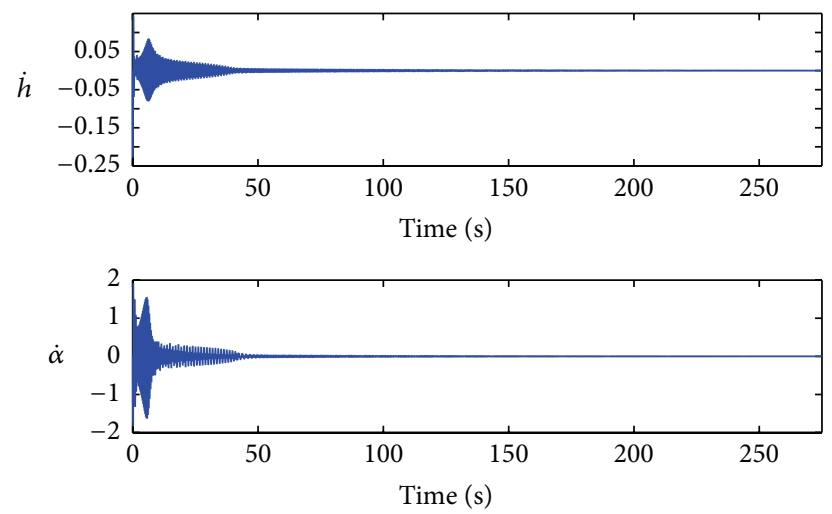

Figure 5: Case 1: $x(0)=[0.02,0.2,0,0]^{T}$; convergence of the regulation error rate for plunging, $\dot{h}(t)$, and pitching, $\dot{\alpha}(t)$.
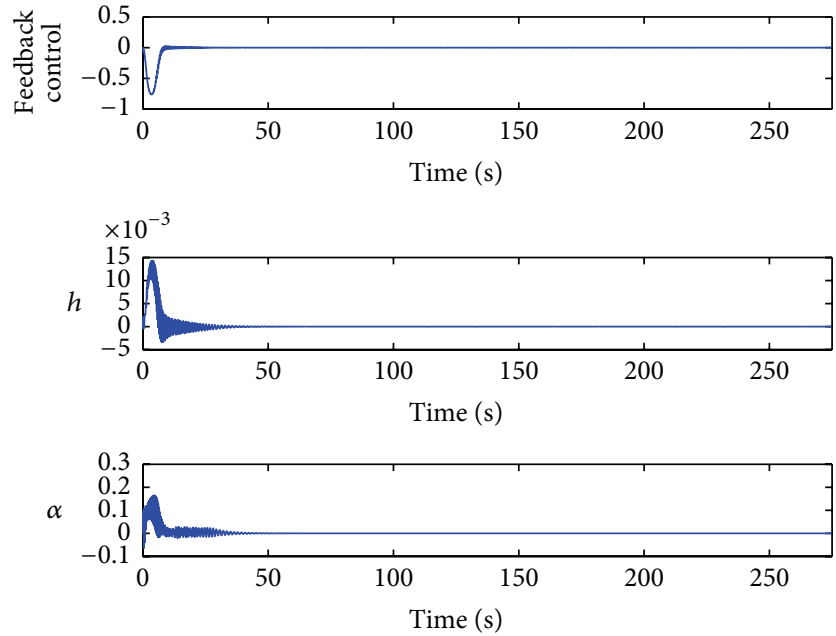

Figure 6: Case 2: $x(0)=[0,0,0.05,0.5]^{T}$; time evolution of the control input, $u(t)$, regulation error for plunging, $h(t)$ in $[\mathrm{m}]$, and regulation error for pitching, $\alpha(t)$ in [rad].
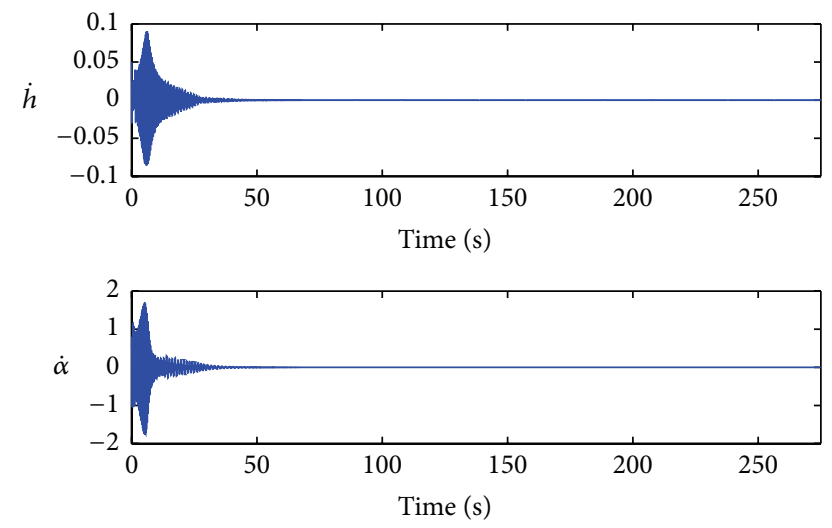

FIgURE 7: Case 2: $x(0)=[0,0,0.05,0.5]^{T}$; convergence of the regulation error rate for plunging, $\dot{h}(t)$, and pitching, $\dot{\alpha}(t)$.
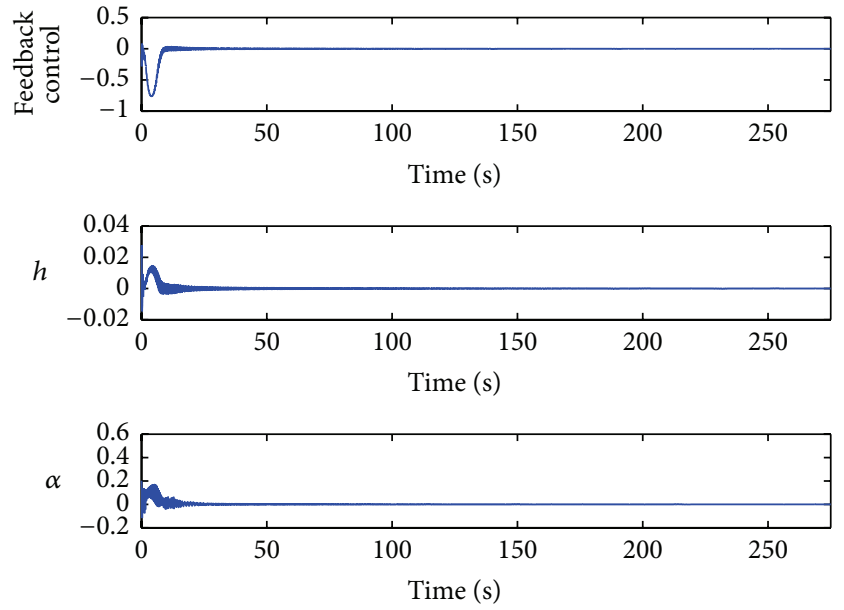

FIGURE 8: Case 3: $x(0)=[0.02,0.2,0.03,0.3]^{T}$; time evolution of the control input, $u(t)$, regulation error for plunging, $h(t)$ in $[\mathrm{m}]$, and regulation error for pitching, $\alpha(t)$ in [rad].
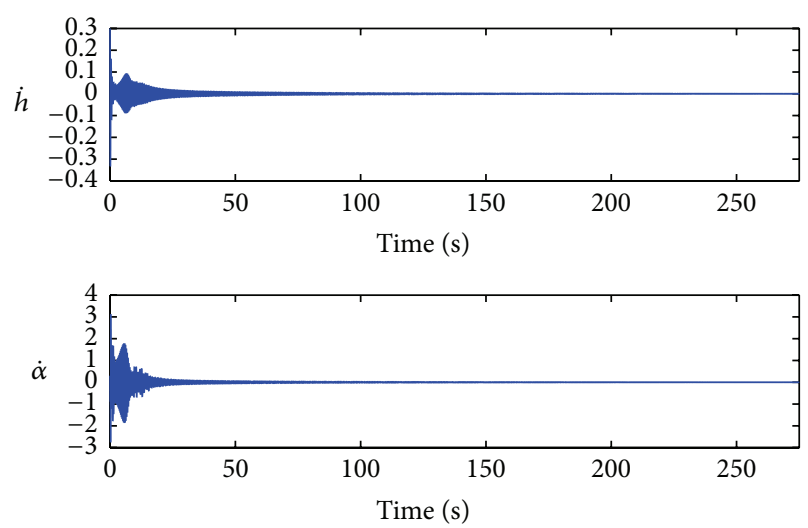

FIGURE 9: Case 3: $x(0)=[0.02,0.2,0.03,0.3]^{T}$; convergence of the regulation error rate for plunging, $\dot{h}(t)$, and pitching, $\dot{\alpha}(t)$.

\section{Conclusion}

In this paper, a sliding mode control law is presented, which achieves asymptotic LCO regulation in UAV wings using SJAs. Moreover, the control law achieves asymptotic regulation of both the pitching and plunging displacements for UAV LCO dynamics in a dual-parallel underactuated form, where a single scalar control signal simultaneously affects both displacements. To achieve the result, a sliding mode control strategy is utilized, which employs a periodic switching function and a novel sliding surface, which is derived based on the nonlinear dynamics of the UAV LCO system. The inherent nonlinearity and parametric uncertainty in the SJA dynamic model is addressed by means of a robust inverse control structure, which utilizes constant feedforward estimates of the uncertain SJA parameters. The use of constant estimates as opposed to time varying adaptive parameter estimates is motivated by the desire to develop a practical LCO regulation method that can be implemented on small UAVs with limited onboard computational resources. A rigorous analysis is presented to prove the theoretical result, 
and numerical simulation results are provided to complement the theoretical development.

\section{Conflict of Interests}

The authors declare that there is no conflict of interests regarding the publication of this paper.

\section{Acknowledgment}

This research is supported by NSF Award number 1335405.

\section{References}

[1] Q. Xia, S. Lei, J. Ma, and S. Zhong, "Numerical study of circular synthetic jets at low Reynolds numbers," International Journal of Heat and Fluid Flow, vol. 50, pp. 456-466, 2014.

[2] K. V. Singh, "Active aeroelastic control with time delay for targeted flutter modes," Aerospace Science and Technology, vol. 43, pp. 281-288, 2015.

[3] Z. Sun, S. Haghighat, H. H. T. Liu, and J. Bai, “Time-domain modeling and control of a wing-section stall flutter," Journal of Sound and Vibration, vol. 340, pp. 221-238, 2015.

[4] N. Ramos-Pedroza, W. MacKunis, and M. Reyhanoglu, "Sliding mode control-based limit cycle oscillation suppression for UAVs using synthetic jet actuators," in Proceedings of the International Workshop on Recent Advances in Sliding Modes (RASM '15), pp. 1-5, Istanbul, Turkey, April 2015.

[5] M. Dardel and F. Bakhtiari-Nejad, "Limit cycle oscillation control of wing with static output feedback control method," Aerospace Science and Technology, vol. 24, no. 1, pp. 147-160, 2013.

[6] M. Ben Chiekh, M. Ferchichi, J.-C. Béra, and M. Michard, "Minimization of time-averaged and unsteady aerodynamic forces on a thick flat plate using synthetic jets," Journal of Fluids and Structures, vol. 54, pp. 522-535, 2015.

[7] Y.-W. Lv, J.-Z. Zhang, Y. Shan, and X.-M. Tan, "Numerical investigation for effects of actuator parameters and excitation frequencies on synthetic jet fluidic characteristics," Sensors and Actuators A: Physical, vol. 219, pp. 100-111, 2014.

[8] T. Persoons, "General reduced-order model to design and operate synthetic jet actuators," AIAA Journal, vol. 50, no. 4, pp. 916-927, 2012.

[9] A. Kourta and C. Leclerc, "Characterization of synthetic jet actuation with application to Ahmed body wake," Sensors and Actuators, A: Physical, vol. 192, pp. 13-26, 2013.

[10] D. Deb, G. Tao, J. O. Burkholder, and D. R. Smith, "Adaptive synthetic jet actuator compensation for a nonlinear aircraft model at low angles of attack," IEEE Transactions on Control Systems Technology, vol. 16, no. 5, pp. 983-995, 2008.

[11] S. H. Kim and C. Kim, "Separation control on NACA23012 using synthetic jet," Aerospace Science and Technology, vol. 13, no. 4-5, pp. 172-182, 2009.

[12] M. Ciuryla, Y. Liu, J. Farnsworth, C. Kwan, and M. Amitay, "Flight control using synthetic jets on a cessna 182 model," AIAA Journal of Aircraft, vol. 44, no. 2, pp. 642-653, 2007.

[13] M. Ben Chiekh, M. Ferchichi, and J.-C. Béra, "Modified flapping jet for increased jet spreading using synthetic jets," International Journal of Heat and Fluid Flow, vol. 32, no. 5, pp. 865-875, 2011.
[14] D. Deb, G. Tao, J. O. Burkholder, and D. R. Smith, "Adaptive compensation control of synthetic jet actuator arrays for airfoil virtual shaping," Journal of Aircraft, vol. 44, no. 2, pp. 616-626, 2007.

[15] M. Krstic, I. Kanellakopoulos, and P. Kokotović, Nonlinear and Adaptive Control Design, Wiley-Interscience, 1995.

[16] G. Tao, Adaptive Control of Systems with Actuator and Sensor Nonlinearities, John Wiley \& Sons, New York, NY, USA, 1996.

[17] W. MacKunis, S. Subramanian, S. Mehta, C. Ton, J. W. Curtis, and M. Reyhanoglu, "Robust nonlinear aircraft tracking control using synthetic jet actuators," in Proceedings of the 52nd IEEE Conference on Decision and Control (CDC '13), pp. 220-225, IEEE, Firenze, Italy, December 2013.

[18] M. R. Elhami and M. F. Narab, "Comparison of SDRE and SMC control approaches for flutter suppression in a nonlinear wing section," in Proceedings of the American Control Conference (ACC '12), pp. 6148-6153, Montreal, Canada, June 2012.

[19] S. V. Drakunov, "Sliding mode control with multiple equilibrium manifolds," Journal of Dynamic Systems, Measurement, and Control, vol. 55, no. 1, pp. 101-108, 1994.

[20] J. J. E. Slotine and W. Li, Applied Nonlinear Control, Prentice Hall, Englewood Cliffs, NJ, USA, 1991. 

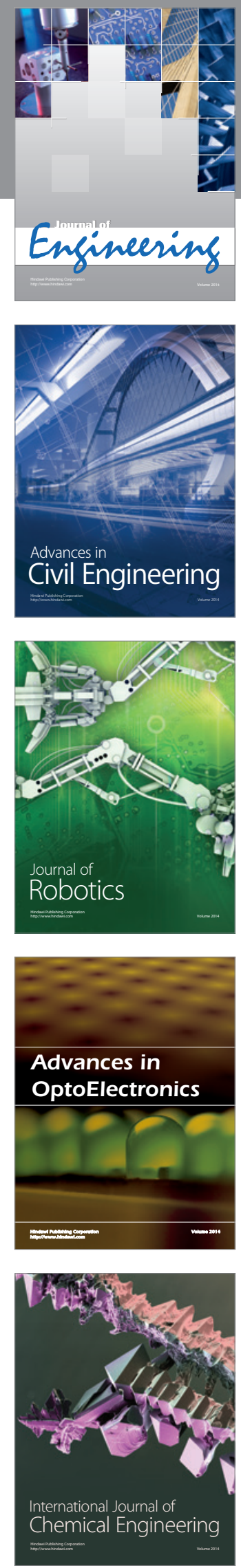

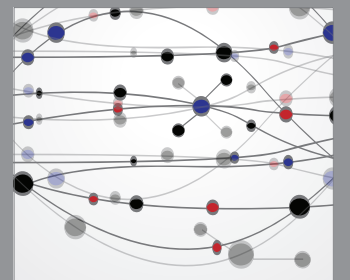

The Scientific World Journal
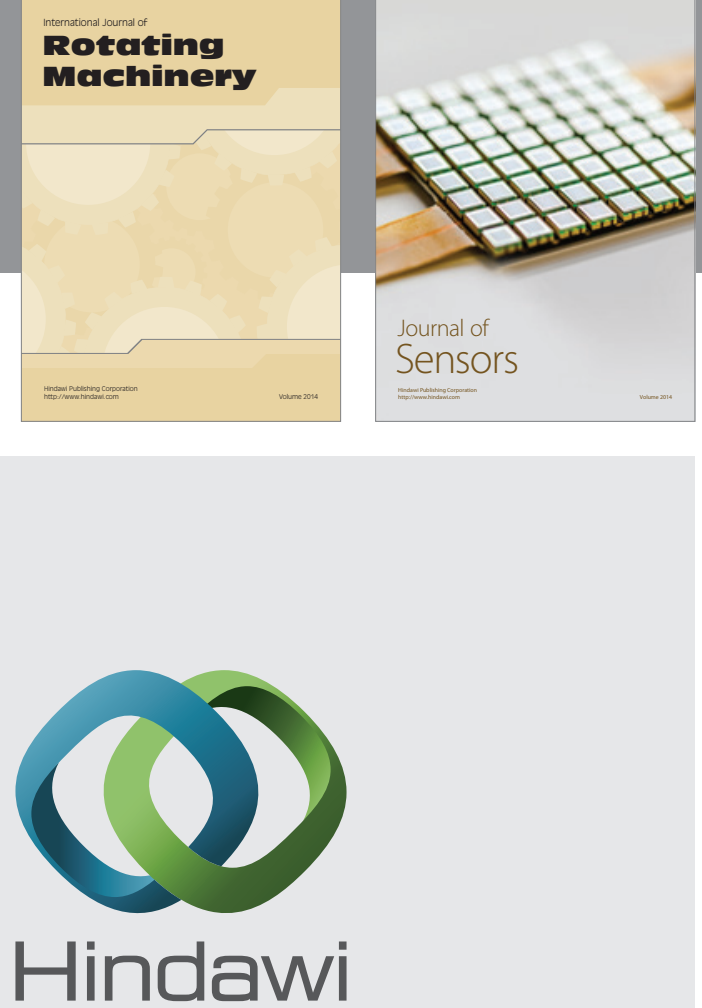

Submit your manuscripts at http://www.hindawi.com
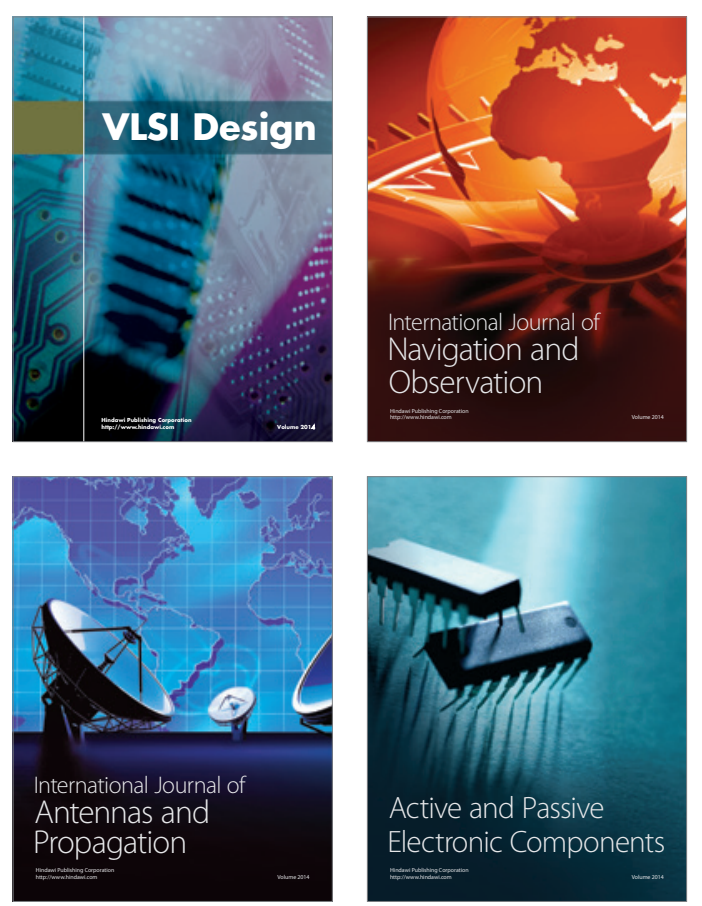
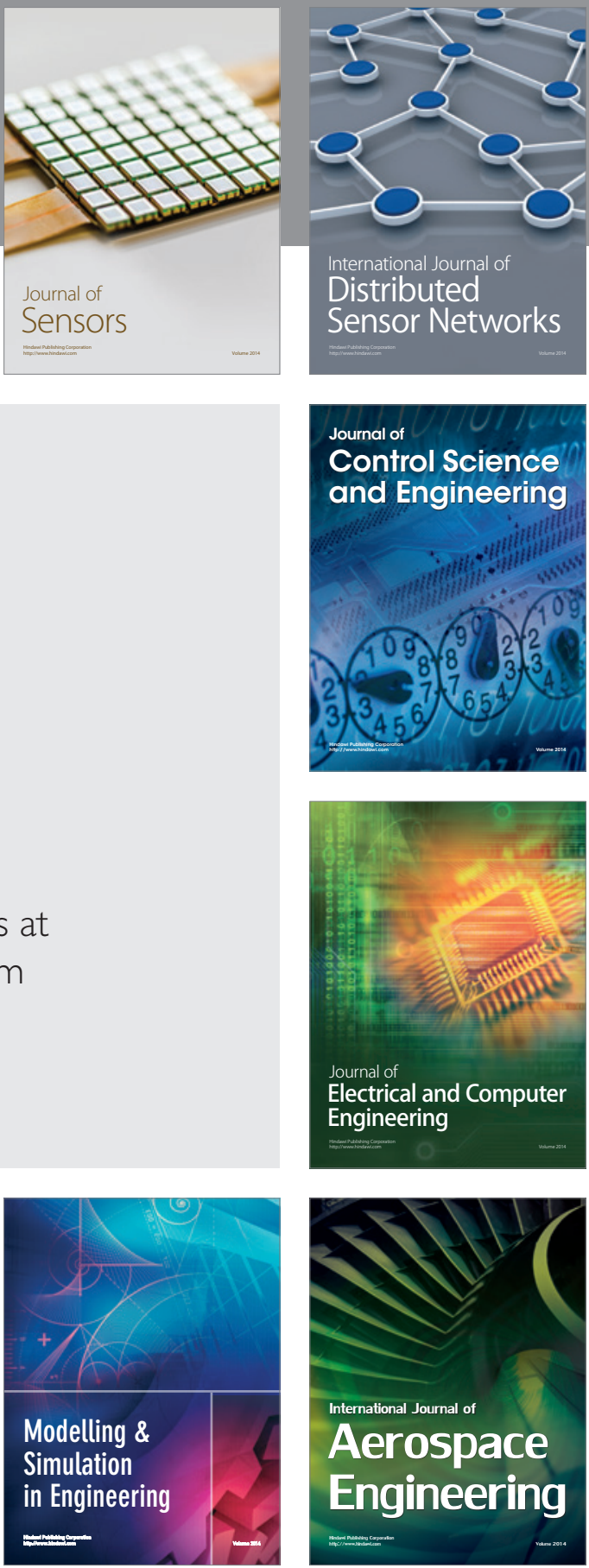

Journal of

Control Science

and Engineering
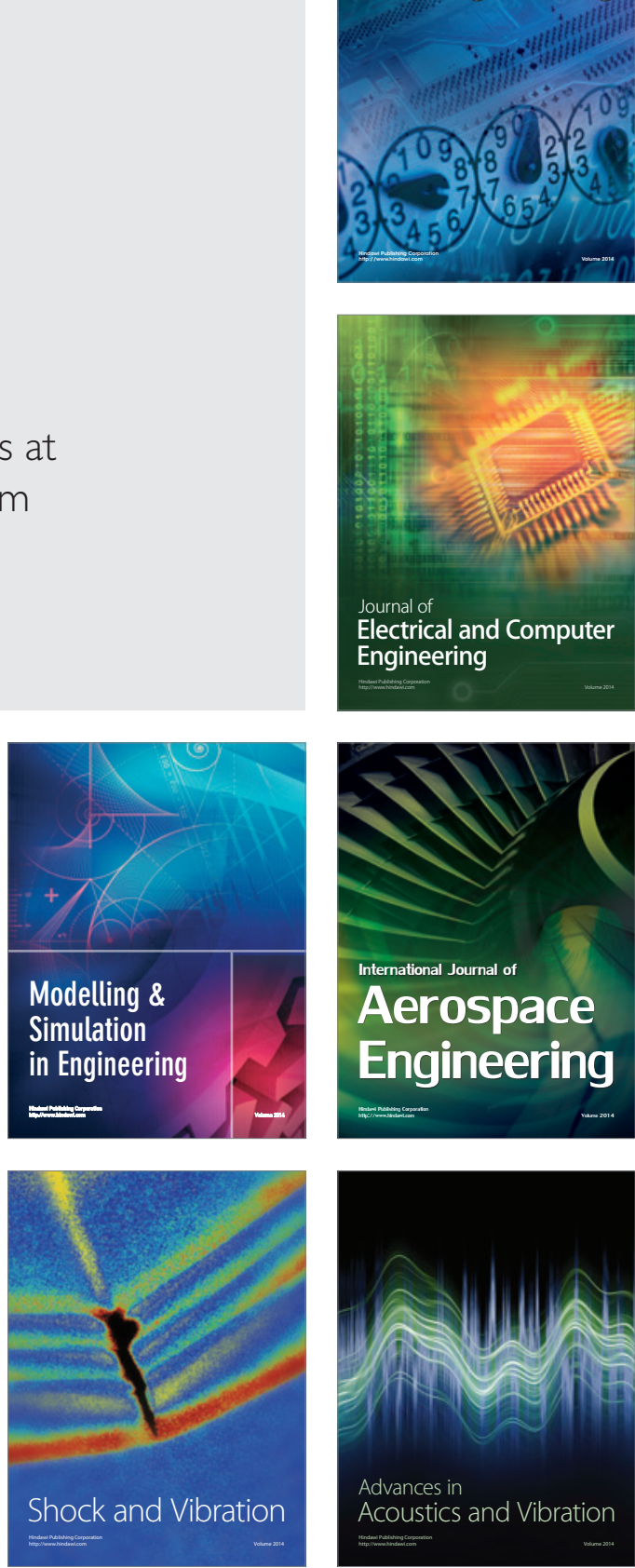